\title{
Oral Health Status and Impact on Oral Health-Related Quality of Life in Children with Thalassemia Major
}

\author{
Nuttaya Phrai-in*, Juntima Noikeaw, Nattaporn Sukprasert, Thitirat Taya and Patcharaphol Samnieng \\ Department of Preventive Dentistry, Faculty of Dentistry, Naresuan University, Phitsanulok, 65000, Thailand \\ *E-mail: sphraiin.01@gmail.com
}

\begin{abstract}
Objective: The purpose of this study was to assess oral health status in children with thalassemia major, and the association of oral health status with oral-health related to quality of life.

Methods: This cross-sectional study was comprised of 81 patients from various parts of lower northern Thailand. The study consisted of an interview and intraoral examination on thalassemia children at the hospital while they were undergoing routine blood transfusions. Data regarding OHI-S, DMFT/dmft and child-OIDP were determined for all the subjects.

Results: The prevalence of oral impacts was high; $82.8 \%$ of all children reported at least one oral impact affecting their daily performance according to the Child-OIDP. The most prevalent impact was difficulty in eating.

Conclusion: The risks of oral disease in thalassemia children remain high which affect their quality of life.

Keywords: oral health, quality of live, thalassemia major
\end{abstract}

\section{Introduction}

Thalassemia is an inherited autosomal recessive blood disorder. The genetic defect results in reduced rate of synthesis of one of the globin chains that form hemoglobin which in turn causes anemia. Thalassemia major (Cooley's anemia) occurs due to striking deficiency in beta chain production of globin in hemoglobin.

Beta Thalassemia major exhibits the most severe clinical symptoms while Thalassemia minor (alpha thalassemia) is mild and clinically asymptomatic. ${ }^{1}$ It is a disease which not only affects patients but also leaves a devastating psychosocial effect on patients' family. ${ }^{1}$

In Southeast Asia, $\alpha$-thalassaemia, $\beta$-thalassaemia, hemoglobin E ( $\mathrm{Hb}$ E) and Hemoglobin Constant Spring ( $\mathrm{Hb} \mathrm{CS})$ are prevalent. The gene frequencies of $\alpha$ thalassaemia reach $30-40 \%$ in Northern Thailand and Laos, $4.5 \%$ in Malaysia and $5 \%$ in the remote island of the Philippines whereas $\beta$-thalassaemia is vary between 1-9\%. $\mathrm{Hb} \mathrm{E}$ is the hallmark of Southeast Asia attaining a frequency of $50-60 \%$ at the junction of Thailand, Laos, and Cambodia. Hb CS gene frequencies vary between 1$8 \% .^{2,3}$

The structural change in oral cavity occurs commonly in thalassemia patients due to maxillary enlargement; such as protrusion of anterior teeth, spacing teeth, deep bite, open bite and varying degree of malocclusion in which further predisposed to dental caries. ${ }^{4}$ Previous studies have reported a higher frequency of periodontal diseases and dental caries in thalassemia patients. ${ }^{5}$

A child's oral health can have a profound impact on eating, smiling, speaking and socializing. Oral conditions; such as dental caries may result in pain. This may lead to consequences on a child's daily life; such as taking time off from school or difficulty eating. The child version of the oral impacts on daily performances (Child-OIDP) is the OHRQoL measure. OHRQoL may be used to analyze the condition-specific impacts on daily performances. ${ }^{6,7}$

Not much information is available on the association of dental caries with Beta thalassemia which affects oral health related quality of life. Therefore, there is a need to assess the oral health status of the children with thalassemia, a disease which poses a major health problem to the society. Thus, the aim of this study was to assess oral health status in children with thalassemia major, and the association of oral health status with oral-health related to quality of life (OHRQoL).

\section{Method}

This cross-sectional study was comprised of 81 patients from various parts of lower northern Thailand. These patients were registered in the Thalassaemia Welfare Association and diagnosed with $\beta$-thalassaemia major. This study is approved by Narasuan University ethical committee. 
The study consisted of an interview and intraoral examination on thalassemia children at the hospital while they were undergoing routine blood transfusions. Data regarding the age, gender and educational status of all the children were recorded. Plane mouth mirror \& explorer were used to examine the oral cavity. Data collection included DMFT index and Oral Hygiene Index-Simplified (OHI-S). DMFT index is for permanent teeth and $\mathrm{dmft}$ index is for primary teeth to record dental caries experience according to the criteria described by the World Health Organization. ${ }^{8}$. Oral hygiene status was recorded using Oral Hygiene Index-Simplified (OHI-S). ${ }^{9}$

A relationship between the quality of life and the oral health is evaluated by the Child-OIDP index (Child-Oral Impact on Daily Performances). In the application of Child-OIDP, the children were asked to record all of the oral-related health problems that they have been experiencing in the past 3 months. Self-reported oral problems were measured by a list of common oral problems that were marked by the children. This was carried out in small groups to reduce share time. Then, the data was collected on the effects of oral problems through face-to-face interviews considering eight common daily performances. These performances are eating, speaking, cleaning mouth, sleeping, emotional status, smiling, studying, and social contact. ${ }^{6}$

Descriptive statistics including mean and standard deviation for OHI-S, DMFT/dmft and child-OIDP were determined for all the subjects who were examined, using SPSS software version 17. A P $<0.05$ was considered to be significant.

\section{Results}

Eighty-one children were participated in this study. There were 37 females $(45.7 \%)$ and 44 males $(54.3 \%)$. Average age of participants was 8.7 years $(\mathrm{SD}=3.7)$ (range $2-15$ years). The thalassemia major was diagnosed in 22 patients at the age of 2-5 years $(27.1 \%), 37$ patients at the age of $6-10$ years $(45.7 \%)$ and 23 patients at the age of $11-$ 15 years $(27.2 \%)$ (Table 1.$)$.

Table 1. Distribution of thalassemia children according to gender and age $(\mathbf{n}=\mathbf{8 1})$

Number of Thalassemia children

\begin{tabular}{ll}
\hline Gender & \\
\hline Female & $37(45.7)$ \\
Male & $44(54.3)$ \\
\hline Age & \\
\hline 2-5 Years & $22(27.1)$ \\
6-10 Years & $37(45.7)$ \\
11-15 Years & $23(27.2)$
\end{tabular}

Table 2 showed oral health status of thalassemia children. Caries prevalence of thalassemia children was 5.0 teeth/person $(\mathrm{SD}= \pm 4.5)$ and OHIS score was $2.28(\mathrm{SD}=$ $0.7)$.
Table 2. Oral health status of thalassemia children

\begin{tabular}{lc}
\hline \multicolumn{1}{c}{ Oral health status } & Mean of thalassemia children \\
\hline Dental caries & $5.0 \pm 4.5$ teeth/person \\
OHIS score & $2.28 \pm 0.7$ \\
\hline
\end{tabular}

The prevalence of oral impacts was high; $82.8 \%$ of all children reported at least one oral impact affecting their daily performance according to the Child-OIDP. The most prevalent impact was difficulty in eating (59\%), following by the impacts on emotional status $(33.6 \%)$, cleaning mouth (33.3\%), smiling (21.3\%), speaking (16.0\%), social contact $(13.2 \%)$, sleeping $(10.5 \%)$ and studying $(9.7 \%)$ respectively were showed in Table 3 .

Table 3. Prevalence of oral impacts on daily performances (Child-OIDP) in thalassemia children

\begin{tabular}{ll}
\hline \multicolumn{1}{c}{ Performance } & $\begin{array}{c}\text { Percentage of children with } \\
\text { Impact on performance }(\boldsymbol{n}=81)\end{array}$ \\
Eating & 59.4 \\
Speaking & 16.0 \\
Cleaning mouth & 33.3 \\
Sleeping & 10.5 \\
Smiling & 21.3 \\
Emotional status & \\
Studying & 9.7 \\
Social contact & \\
At least on of above & 82.8 \\
\hline
\end{tabular}

\section{Discussion}

This study was probably among first attempts in Thailand to compare the prevalence of dental caries and oral hygiene index in beta thalassemia patients. This study found that the patients with Beta thalassemia $(5.0 \pm 4.5$ teeth/person) had higher caries experience than the normal children (4.0 teeth/person in 5 years old). ${ }^{10}$ Level of dental caries may be explained on the basis of chronic nature of thalassemia. Patients preoccupied with their major life threatening condition, therefore neglecting a basic preventive dental care. ${ }^{11}$ Another reason could be because of the increased sensitivity to local factors (oral hygiene, tooth brushing habit and frequency of sugar intake). ${ }^{12}$

The present study also showed that prevalence of dental caries was significantly higher in thalassemia patients than in age matched healthy controls, suggesting a need for restorative dental care in thalassemia children. Certain structural changes in oral cavity that due to maxillary enlargement result in protrusion of anterior teeth, increased space between teeth, over-bite or open-bite and 
varying degrees of malocclusion, which further predispose to caries. ${ }^{5}$

This study showed that thalassemia child had poor oral hygiene consistency with previous study. Results from the examination of thalassemia patients and healthy controls suggested variations in their oral hygiene as measured by OHI-S. OHI-S is founded to be increased in Thalassemia patients. ${ }^{13}$ The higher caries prevalence can be attributed to the poor oral hygiene, improper dietary habits, lack of dental knowledge, poor motivation, reduced salivary urea concentration. This could also be attributed to parental overprotection or negligence in these systemically ill patients. $^{13}$

This study found that eating was the most important aspect of OHRQoL of children. Difficulty with eating due to oral problems was the most common impact (59.4\%), and lead to more severe oral impacts on children's quality of life than impacts on other performances. The finding that eating was the most common performance affected is similar to all studies using the OIDP in all age groups. ${ }^{14,15}$, ${ }^{16}$ Oral conditions such as dental caries and malocclusions affect self-esteem, the ability to chew and speak, and may be associated with absenteeism from school and psychological problems. ${ }^{17,}{ }^{18}$ However, our study has some limitations. Sample size of population in this study was small and only from just one hospital. Secondly, the crosssectional study cannot relate an inference about casual relationships. Hence further studies are required in this field with suggestions of increasing the sample sizes and considerably longer durations to validate our findings.

\section{Conclusions}

The risks of oral disease in thalassemia children remain high. Prevention against oral diseases is very important for improving a life expectancy of these patients and the role of good oral status in better quality of life. Emphasis to educate such group of individuals and their parents in the prevention of dental caries and oral hygiene should be considered and oral health education must be given to beta thalassemia child right from the detection of disease to prevent more serious dental diseases.

\section{References}

1. Al-Wahadni AM, Taani DQ, Al Omari MO. Dental diseases in subjects with beta thalassemia major. Community Dent Oral Epidemiol. 2002; 30:418-422.
2. Wasi P. Hemoglobinopathies in Southeast Asia. In: Bowman JE, editor. Distribution and evolution of hemoglobin and globin Loci. New York: Elsevier; 1983. p. 179-203.

3. Fucharoen S, Winichagoon P. Hemoglobinopathies in Southeast Asia. Hemoglobin. 1987; 11 : 65-8.

4. SK Kataria, M Arora, A Dadhich, KR Kataria. Orodental complications and orofacial menifestation in children and adolescents with thalassaemia major of western Rajasthan population: a comparative study. Int J Biol Med Res. 2012;3:1816-1819.

5. Al-Wahadni AM, Taani DQ, Al-Omari MO.Dental diseases in subjects with beta thalassemia major. Community Dent Oral Epidemiol. 2002; 30: 418-422.

6. Gherunpong S, Tsakos G, Sheiham A. Developing and evaluating an oral health-related quality of life index for children; the CHILD-OIDP. Community Dent Health. 2004; 21: 161-169.

7. Sheiham A, Tsakos G. Oral health needs assessments. In: Pine C, Harris R (eds). Community Oral Health. London: Quintessence Publishing, 2007: 59-79.

8. WHO. Oral health surveys: basic methods. (5th ed.). 2013

9. Greene and Vermillion. Simplified Oral Hygiene Index. Sweden: Malmö University; 1964.

10. Dental division, Ministry of Health. 7th National oral health surveys of Thailand. Available from http://dental.anamai.moph.go.th/elderly/academic/f ull99.pdf

11. E Guzeldemir, HU Toygar, C Boga, U Cilasun. Dental and periodontal health status of subjects with sickle cell disease. J Dent Sci. 2011;6:227-234.

12. $\mathrm{R}$ Veena. Dental caries and periodontal health status in thalassemic major patients. Karnataka, Bangalore Rajiv Gandhi University and Health Sciences. 2006:1-116.

13. Vijaya Dhote. Evaluation of oral hygiene status and salivary biochemistry of patients with Thalassemia major: A clinical study. Journal of Dental and Medical Sciences. 2015; 14(12):98-102.

14. Jokovic A, Locker D, Stephens M, Kenny D, Tompson B, Guyatt G. Validity and reliability of a questionnaire for measuring child oral-health-related quality of life. J Dent Res. 2002;81:459- 463 .

15. Adulyanon S, Vourapukjaru J, Sheiham A. Oral impacts affecting daily performance in a low dental disease Thai population. Community Dent Oral Epidemiol. 1996;24:385-389.

16. Srisilapanan P, Sheiham A. The prevalence of dental impacts on daily performances in older people in Northern Thailand. Gerodontology. 2001;18:102-108.

17. K.G. Peres, A.M. Cascaes, A.T. Leão, M.I. Côrtes, M.V. Vettore Sociodemographic and clinical aspects of quality of life related to oral health in adolescents. Rev Saude Publica. 2013; 47 (3):19-28.

18. H.S. Schuch, F. Dos Santos Costa, D.D. Torriani, F.F Demarco, M.L. Goettems Oral health-related quality of life of schoolchildren: impact of clinical and psychosocial variables. Int J Paediatr Dent. 2015; 25 (5): 358-365 